\title{
Effets de l'incorporation de pulpe de betterave dans les aliments du porcelet et du porc en croissance : performances de croissance, digestibilité et composition corporelle
}

\author{
R Lizardo *, J Peiniau, Y Lebreton, A Aumaitre \\ Station de recherches porcines, Inra, 35590 Saint-Gilles, France
}

(Reçu le 3 janvier 1996 ; accepté le 6 novembre 1996)

\begin{abstract}
Summary - Consequences of the inclusion of sugar beet pulp in diets for early weaned and growing pigs. The effect of extrusion of wheat and of the inclusion of $6 \%$ of sugar beet pulp in a pelleted starter on the performance of the weaned piglet has been studied in two experiments. In the first experiment, 80 crossbred castrated male and female piglets weaned at 21 days of age raised individually were used in a $2 \times 2$ factorial arrangement to compare their performance between weaning and $25 \mathrm{~kg}$ live weight. Average feed intake was similar between treatments, but extrusion of wheat had no significant effect on growth performance and feed conversion ratio. The association of normal wheat with sugar beet pulp improved significantly growth rate $(P<0.02)$ and feed conversion ratio $(P<0.06)$, in particular during the period following immediatly weaning, ie, $193 \mathrm{vs} 156 \mathrm{~g} /$ day and $1.93 \mathrm{vs} 2.32$, respectively. In the second experiment, 32 crossbred castrated piglets were used to evaluate faecal, then ileal digestibilities of dietary components. Extrusion did not improve digestibility. Ileal digestibility but not faecal digestibility of dry matter and energy was reduced $(P<0.001)$ by the inclusion of sugar beet pulp in the diet. Post-ileal digestibility of all dietary components was higher in animals fed fibrous diet. During the growing-finishing period between 25 and $100 \mathrm{~kg}$ live weight, the inclusion of a similar level of $6 \%$ of sugar beet pulp in a normal wheat based diet was tested. Piglets of the first experiment were randomly affected to one of the two diets taking into account their previous diet, sex and weight. The average daily gain for the whole period was slighly but not significantly reduced and feed conversion ratio was unchanged in animals receiving sugar beet pulp. However, feed conversion ratio expressed as digestible energy per kg of body weight gain was reduced in animals receiving beet pulp in their diet. At slaughter, the percentage of lean meat was improved from 52.8 to $54.0 \%$ $(P<0.05)$ as a consequence of a linear decrease in the backfat thickness $(P<0.05)$ of carcasses of animals receiving sugar beet pulp. No difference in the weight of lean cuts was observed but the use of sugar beet pulp decreased significantly the weight of fat cuts $(P<0.01)$. It was suggested that for a similar nitrogen retention a reduction in the digestible energy intake reduces the deposition of fatty tissues but the total amount of lean meat in the carcass was not affected by the inclusion of sugar beet in the diet.
\end{abstract}

pig / sugar beet pulp / weaning / growth / digestibility / body composition

\footnotetext{
* Correspondance et tirés à part.
} 
Résumé - L'effet de l'incorporation de $6 \%$ de pulpe de betterave a été comparé à un régime de sevrage à base de blé ou de blé extrudé chez le porcelet sevré à $21 \mathrm{j}$ et jusqu'à $25 \mathrm{~kg}$ de poids vif. L'extrusion ne modifie pas les performances zootechniques, mais l'addition de pulpe de blé améliore significativement le gain moyen quotidien $(p<0,02)$ ainsi que l'indice de consommation $(p<0,06)$ des porcelets au cours du premier âge. La digestibilité fécale (28-35 j) des composants ne montre pas de différence entre régimes. Au contraire, la digestibilité iléale (42-49j) de la matière sèche, de l'énergie et la rétention azotée $(p<0,001)$ sont réduites significativement par l'incorporation de la pulpe. Cependant, le calcul de la digestibilité post-iléale montre une récupération des nutriments énergétiques et azotés $(p<0,05)$ dans le gros intestin. L'addition d'un même taux de $6 \%$ de pulpe de betterave à un régime à base de blé en phase de croissance et de finition n'entraîne pas de différence significative ni sur la croissance journalière ni sur l'indice de consommation ; elle réduit, en revanche, l'indice de consommation énergétique. À l'abattage, on observe une réduction de l'épaisseur du lard dorsal et une augmentation du pourcentage de muscle de la carcasse chez les animaux ayant reçu le régime additionné de pulpe $(p<0,05)$. Les données de découpe confirment une diminution du poids de la panne, de la bardière et de la poitrine $(p<0,01)$, alors que le poids des pièces maigres est identique.

porc / pulpe de betterave / sevrage / croissance / digestibilité / composition corporelle

\section{INTRODUCTION}

La capacité digestive du porcelet n'est pas suffisante pour utiliser pleinement l'aliment sec concentré distribué après un sevrage brutal pratiqué entre 21 et $28 \mathrm{j}$ (Kelly et al, 1990). Ainsi, l'activité des enzymes pancréatiques est encore faible à cet âge (Peiniau et al, 1996) et, si l'estomac et l'intestin grêle sont fortement sollicités au cours de l'allaitement, le gros intestin n'est pas suffisamment développé et l'activité de la flore microbienne est très réduite (Bolduan et al, 1988). Il en résulte des perturbations dans le rythme d'ingestion de l'aliment et des intolérances digestives qui se traduisent par des diarrhées de post-sevrage variables en durée et en gravité. Pour pallier ces troubles digestifs, l'addition de substances antimicrobiennes, antibiotiques, probiotiques ou acides organiques est pratiquée sans être complètement satisfaisante (Eidelsburger et al, 1992). En revanche, l'incorporation d'amidon très digestible apporté par les céréales extrudées (Björck et Asp, 1983) pourrait conduire à une adaptation plus rapide à l'aliment concentré. À l'opposé, l'utilisation de matières premières riches en polysaccharides non amylacés (PNA) a été également proposée pour développer la fonction de digestion dans le gros intestin (Partridge et Gill, 1993). L'addition de pulpe de betterave, riche en substances pectiques facilement fermentescibles, aux aliments de sevrage (Longland et al, 1994) a conduit à une amélioration de l'état sanitaire des porcelets sans affecter les performances de croissance (Edwards et al, 1991). De tels résultats peuvent être expliqués par la forte digestibilité fécale des PNA de la pulpe de betterave chez le porcelet dès $32 \mathrm{j}$ d'âge (Low et al, 1990) et par une digestibilité élevée de la fraction fibre au détergent neutre (NDF) au niveau iléal (Laplace et al, 1994) et au niveau fécal (Shi et Noblet, 1994a ; Chabeauti et al, 1991) chez le porc en croissance. L'association entre les PNA fermentescibles de la pulpe et les céréales extrudées dans la ration semble appropriée aux besoins physiologiques du porcelet, mais son effet sur les performances zootechniques et digestives n'a jamais été étudié. Chez le porc en croissance-finition alimenté de façon restreinte, l'incorporation de fibres dans les régimes réduit leur teneur en énergie digestible (Henry, 1976 ; Noblet et al, 1989), 
réduisant la vitesse de croissance et, dans le cas de la pulpe, l'adiposité de la carcasse (Chauvel et al, 1975). L'absence d'effet de la pulpe de betterave sur l'utilisation digestive et métabolique de l'azote (Longland et al, 1994) peut encore expliquer ce résultat. L'objectif de ce travail est d'abord de mesurer les effets zootechniques et digestifs consécutifs à l'incorporation de pulpe de betterave et de blé extrudé dans les aliments pour le porcelet. L'expérience a été poursuivie en période de croissance et de finition afin de vérifier l'effet de l'addition de la pulpe de betterave dans la ration sur les performances de croissance et sur la composition corporelle à l'abattage.

\section{MATÉRIEL ET MÉTHODES}

\section{Animaux, aliments et dispositifs expérimentaux}

Deux expériences ont été réalisées : une première expérience zootechnique a porté sur des porcelets entre le sevrage et $25 \mathrm{~kg}$ de poids vif (PV) et poursuivie chez les mêmes animaux en croissance-finition et jusqu'à l'abattage ; une seconde expérience a consisté à mesurer la digestibilité fécale, puis iléale, chez le porcelet aussi tôt que possible après le sevrage.

Dans l'expérience zootechnique, ont été utilisés 40 porcelets mâles castrés et 40 porcelets femelles, croisés Piétrain $\times$ Large White, sevrés à $21 \pm 2 \mathrm{j}$, et logés individuellement dans un bâtiment à ambiance contrôlée $\left(25 \pm 1{ }^{\circ} \mathrm{C}\right)$. Les animaux sont affectés selon un schéma factoriel $(2 \times 2)$ où les traitements correspondent aux régimes à tester (tableau $\mathrm{I}$ ) à base de blé normal (BN) ou de blé extrudé (BE), additionnés ou non de $6 \%$ de pulpe de betterave (BNP et BEP). Vingt blocs de quatre porcelets de la même portée et de poids identique ont été constitués avec distribution intrabloc au hasard. Des aliments adaptés au premier âge ( 21 à $42 \mathrm{j}$ ) et au deuxième âge ( $42 \mathrm{j}$ à $25 \mathrm{~kg}$ PV, environ) des porcelets ont été formulés, en accord avec les recommandations en énergie, protéine et principaux acides aminés essentiels (Inra, 1989). La même quantité de blé a été incorporée et on a tenté de conserver une teneur en énergie brute (EB) et en protéine brute (PB) identique dans tous les régimes. Le blé a été extrudé à $150^{\circ} \mathrm{C}$ avec addition de vapeur à 3 bar $\mathrm{cm}^{-2}$ dans un extrudeur bivis (Incarblé, Dievet Nutrition, France). La pulpe de betterave contient $11,6 \%$ de PB, $45,5 \%$ de NDF, $23,2 \%$ de fibre au détergent acide (ADF) et 17,2 MJ d'EB par kilo de matière sèche (MS). L'eau et l'aliment granulé (2,5 $\mathrm{mm}$ de diamètre) ont été distribués à volonté et la consommation individuelle d'aliment a été mesurée et rapportée en moyenne sur les deux périodes successives de 21 à $42 \mathrm{j}$ et de $42 \mathrm{j}$ à $25 \mathrm{~kg}$ de PV.

Pendant les phases de croissance (25 à $60 \mathrm{~kg} \mathrm{PV}$ ) et de finition ( 60 à $100 \mathrm{~kg} \mathrm{PV}$ ), deux régimes à base de $\mathrm{BN}$ ou de $\mathrm{BNP}$ (tableau $\mathrm{I}$ ) ont été comparés. Après avoir atteint au minimum $25 \mathrm{~kg}$ de PV, les animaux ont été immédiatement répartis de façon équilibrée dans chacun des deux traitements à tester en tenant compte du régime précédent, du sexe et du poids. Les animaux ont été logés individuellement et l'aliment granulé $(4,5 \mathrm{~mm}$ de diamètre) a été fourni selon un plan de distribution journalière quel que soit le sexe des animaux, avec des augmentations graduelles, soit $1,5 \mathrm{~kg}$, aux alentours de $25 \mathrm{~kg}$ de $\mathrm{PV}$, avec un maximum de $2,9 \mathrm{~kg}$, à partir de $75 \mathrm{~kg}$ de PV et jusqu'à l'abattage (Bourdon et Henry, 1985).

La digestibilité fécale et iléale des principaux composants de la ration a été étudiée, chez le porcelet, grâce à un schéma expérimental identique (factoriel $2 \times 2$ ). Trente-deux porcelets mâles castrés Piétrain $\times$ Large White ont été utilisés dès le sevrage. Après 1 semaine d'adaptation aux régimes, une collecte séparée des fèces et de l'urine a été effectuée durant $7 \mathrm{j}$. Ensuite, les animaux ont subi une anastomose iléorectale et, après 1 semaine de récupération, on a effectué 1 semaine de collecte de l'urine et des effluents iléaux. Les animaux ont été logés individuellement en cages à métabolisme dans un bâtiment à ambiance contrôlée. Des régimes identiques à ceux utilisés pour l'expérience zootechnique ont été testés et la quantité d'aliment allouée correspond à environ $30 \mathrm{~g}$ par kilo de $\mathrm{PV}$ par jour. Cependant, les aliments distribués pendant la semaine de mesure de la digestibilité iléale ont été supplémentés par un mélange de minéraux renforcé principalement en sodium et potassium et en vitamines (Lizardo et al, 1995), de façon à pallier une carence éventuelle provoquée par l'absence de fonctionnement du gros intestin. La digestibilité post-iléale a été estimée à partir des mesures effectuées successivement sur les mêmes animaux selon la méthode proposée par Drochner (1984) d'utiliser la valeur de la digestibilité 


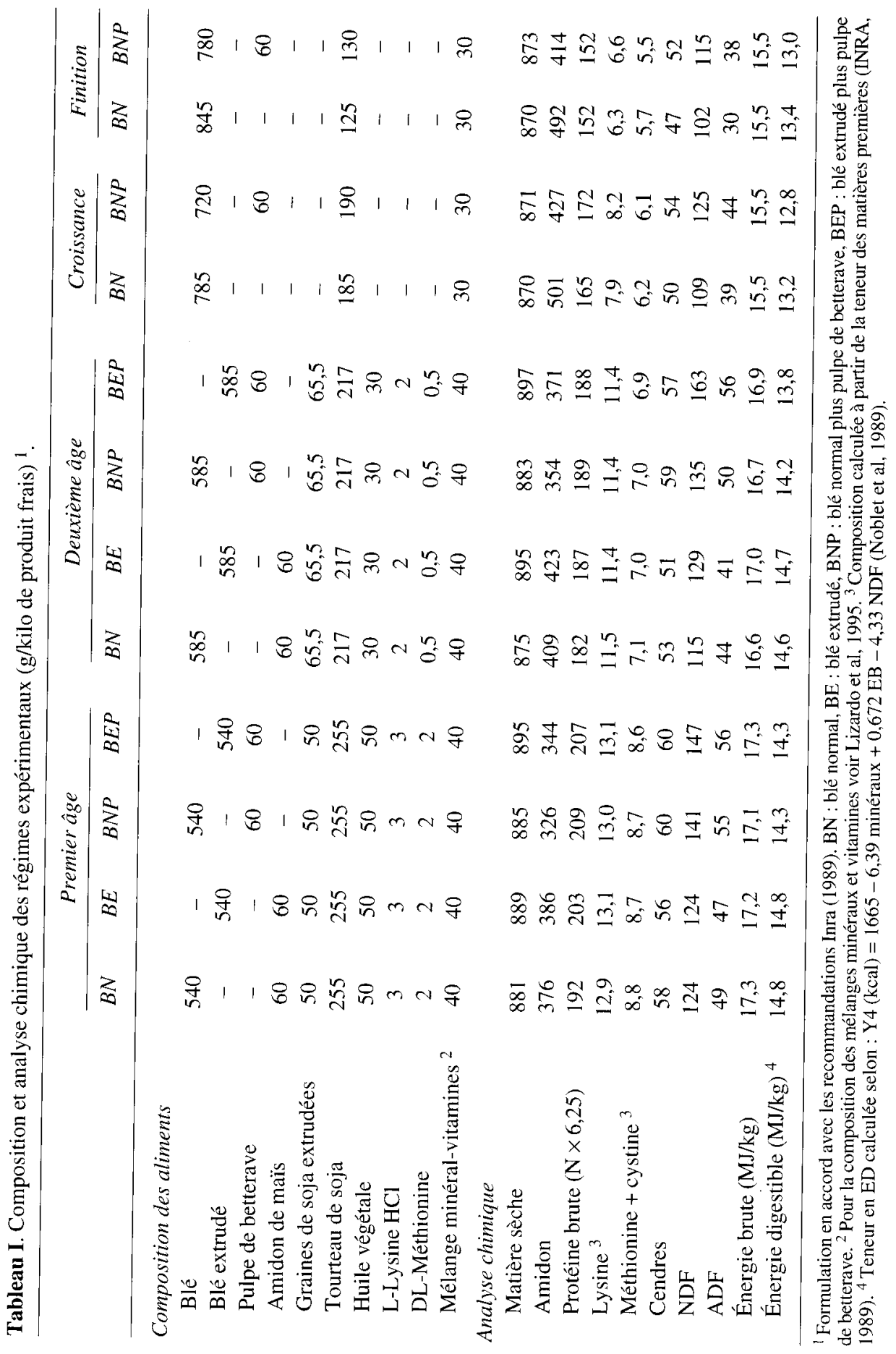


iléale des principaux composants de la ration pour estimer les quantités théoriques arrivant dans le gros intestin chez le même porcelet, lorsque son tube digestif est intact.

\section{Mesures}

Les animaux ont été pesés toutes les semaines et la mesure de l'épaisseur du tissu adipeux souscutané sur l'animal vivant a été effectuée à 60 et $90 \mathrm{~kg}$ de $\mathrm{PV}$ avec un appareil à ultrasons (Toshiba Sonolayer-L, Japon). Les mesures ont été prises au niveau de l'épaule, du dos (dernière côte) et du rein (fessier moyen) à $4 \mathrm{~cm}$ de chaque coté de la ligne longitudinale du dos de l'animal. Les animaux ayant atteint un PV minimal de $98 \mathrm{~kg}$ ont été abattus, après avoir subi un jeûne de 24 h. Après l'éviscération, les organes digestifs, estomac, intestin grêle et gros intestin, ont été pesés avant et après avoir été vidés, de façon à mesurer le poids de l'organe lui-même et de son contenu. Sur la chaine d'abattage, on a procédé à la pesée de la carcasse chaude avec la tête et aux mesures de l'épaisseur de la bardière (X2, $\mathrm{X} 4$; $\mathrm{mm}$ ) et du muscle Longissimus dorsi (X5; mm) prises latéralement avec un appareil optique FatO-Meater (FOM, SFK, Copenhague, Danemark), soit respectivement, $\mathrm{X} 2$, entre la troisième et la quatrième vertèbre lombaire, à $8 \mathrm{~cm}$ de la ligne moyenne de fente de la carcasse et X4, X5, entre les troisième et quatrième dernières côtes, à $6 \mathrm{~cm}$ de la même ligne moyenne (Desmoulin et al, 1988). Ces mesures ont été utilisées pour le calcul du pourcentage de muscle de la carcasse $(\mathrm{Y} 1=57,399-0,33 \mathrm{X} 2-0,441 \mathrm{X} 4+0,193 \times 5$; CCE directive 50, 1989). Environ $24 \mathrm{~h}$ après ressuage en chambre froide à $5^{\circ} \mathrm{C}$, on a procédé à la pesée de la demi-carcasse froide et à la découpe de 24 demi-carcasses gauches selon la découpe parisienne normalisée, en assurant une répartition des animaux en nombre équivalent selon le sexe et le type d'aliment distribué pendant la période de croissance-finition. Le poids en kilo du tissu maigre et du tissu gras de la demi-carcasse a été calculé respectivement à partir des équations suivantes :

Y2 $(\mathrm{kg})=296,9-201,0$ demi-carcasse froide $(\mathrm{kg})+1,395$ longe $(\mathrm{g})+1,144$ jambon $(\mathrm{g})$;

$\mathrm{Y} 3(\mathrm{~kg})=-816+1,63$ bardière $(\mathrm{g})+1,43$ panne (g) $+0,52$ poitrine $(\mathrm{g})$

(Desmoulin et al, 1988).

\section{Analyses de laboratoire et calculs statistiques}

Au laboratoire, les analyses classiques de la teneur en MS, EB, PB $(N \times 6,25)$, cendres (MM) et amidon des régimes (AOAC, 1990) ont été effectuées. Les fibres NDF et ADF ont été déterminées par la méthode de Van Soest et Wine (1967). Les teneurs en lysine et acides aminés soufrés totaux ont été calculées à partir de la teneur moyenne des matières premières incorporées dans les régimes (Inra, 1989). La teneur en énergie digestible (ED) des aliments a été calculée à partir d'une des équations de régression proposées par Noblet et al (1989):

$\mathrm{Y} 4(\mathrm{kcal})=1665-6,39 \mathrm{MM}(\mathrm{g})+0,672 \mathrm{~EB}$ (kcal) - 4,33 NDF (g)

Toutes les données ont été analysées statistiquement par analyse de variance, par la procédure GLM (General linear models) du programme Sas (SAS, 1990). Pour les différentes variables mesurées pendant la phase porcelet, ont été retenus comme effets : l'incorporation de la pulpe de betterave $(\mathrm{Pb})$, l'extrusion du blé (Ex) et l'interaction entre les deux $(\mathrm{Pb} * \mathrm{Ex})$. L'effet sexe a été retiré du modèle car il n'était pas significatif. En période de croissance, de finition et à l'abattage, le régime et le sexe des animaux ont été pris comme effets ; l'interaction régime* sexe n'étant pas significative, a été retirée. L'effet résiduel du traitement lors de la phase postsevrage sur les performances de croissance, finition et de composition corporelle a été testé et enlevé du modèle car il n'était pas significatif. En raison de la mort de trois animaux pendant l'expérience zootechnique, les moyennes ont été ajustées et comparées par un test de StudentNewman-Keuls. Les valeurs de la probabilité sont rapportées dans les tableaux de résultats.

\section{RÉSULTATS}

\section{Performances de croissance}

Chez les porcelets (tableau II), une interaction $(p<0,01)$ s'observe pour la vitesse de croissance et pour l'indice de consommation pendant la période totale (sevrage, $25 \mathrm{~kg}$ PV). En fait, ce sont les régimes contenant soit du BNP, soit du BE, qui permettent les meilleures performances. Pour les deux 
Tableau II. Effet de l'extrusion du blé et de l'addition de pulpe de betterave sur les résultats de croissance des porcelets entre le sevrage et environ $25 \mathrm{~kg}$ de PV.

\begin{tabular}{|c|c|c|c|c|c|c|c|c|}
\hline & \multicolumn{4}{|c|}{ Régimes } & \multicolumn{4}{|c|}{ Analyse statistique ${ }^{I}$} \\
\hline & $B N$ & $B E$ & $B N P$ & $B E P$ & $P b$ & Ex & $P b^{*} E x$ & ETR \\
\hline \multicolumn{9}{|c|}{ Période totale : 21 jà $25 \mathrm{~kg} P V$} \\
\hline Poids initial $(\mathrm{kg})$ & 6,5 & 6,6 & 6,5 & 6,5 & 0,74 & 0,97 & 0,90 & 0,6 \\
\hline Poids final $(\mathrm{kg})$ & 27,5 & 28,0 & 28,4 & 27,8 & 0,43 & 0,86 & 0,17 & 1,8 \\
\hline Durée (j) & 52 & 51 & 50 & 52 & 0,85 & 0,52 & 0,12 & 3 \\
\hline Aliment ingéré (g/jour) & 733 & 724 & 752 & 729 & 0,27 & 0,15 & 0,52 & 50 \\
\hline Gain de poids (g/jour) & 407 & 423 & 437 & 410 & 0,29 & 0,50 & 0,01 & 36 \\
\hline Indice de consommation & 1,81 & 1,72 & 1,73 & 1,79 & 0,88 & 0,61 & 0,01 & 0,13 \\
\hline \multicolumn{9}{|l|}{ Premier âge : 21 à $42 j$} \\
\hline Aliment ingéré (g/jour) & 338 & 337 & 344 & 335 & 0,85 & 0,68 & 0,73 & 53 \\
\hline Gain de poids (g/jour) & $156 b$ & $164 b$ & $193 a$ & $164 b$ & 0,02 & 0,21 & 0,03 & 36 \\
\hline Indice de consommation & 2,32 & 2,18 & 1,91 & 2,16 & 0,06 & 0,61 & 0,08 & 0,48 \\
\hline \multicolumn{9}{|c|}{ Deuxième âge : 42 jà $25 \mathrm{~kg} P V$} \\
\hline Aliment ingéré (g/jour) & 1008 & 999 & 1047 & 1000 & 0,22 & 0,09 & 0,26 & 72 \\
\hline Gain de poids (g/jour) & 584 & 608 & 619 & 583 & 0,70 & 0.66 & 0,03 & 59 \\
\hline Indice de consommation & 1,74 & 1,66 & 1,70 & 1,73 & 0,46 & 0,40 & 0,07 & 0,14 \\
\hline
\end{tabular}

BN : blé normal, BE : blé extrudé, BNP : blé normal plus pulpe de hetterave, BEP : blé extrudé plus pulpe de betterave. ${ }^{\prime}$ Valeurs des moyennes ajustées et de la probabilité $; \mathrm{Pb}:$ pulpe de betterave : Ex : extrusion : $\mathbf{P b} *$ Ex : interaction pulpe de bettcrave*extrusion du blé : ETR : écart type résiduel du modèle ; a, b, c correspondent à la comparaison multiple des moyennes et les valeurs qui ne sont pas affectées d'une même lettre sont significativement différentes à $p<0,05$ par le test de Student-Newman-Keuls.

périodes d'âge séparément, l'interaction significative entre la $\mathrm{Pb}$ et l'Ex $(p<0,03)$ s'observe aussi pour le gain de poids et la même tendance apparaît pour l'indice de consommation $(p<0,08)$. On constate également qu'au cours du premier âge, l'addition de $\mathrm{Pb}$ au régime $\mathrm{BN}$ permet une vitesse de croissance supérieure $(p<0,02)$, atteignant une différence maximale de $22 \%$ entre les régimes extrêmes. La quantité d'aliment ingérée ne varie pas entre les régimes, ce qui entraîne un effet améliorateur de l'introduction de la pulpe sur l'indice de consommation $(p<0,06)$. Au deuxième âge, les différences de croissance se maintiennent mais la variabilité plus importante des résultats ne permet pas de montrer une différence significative. On observe seulement une tendance à la réduction de la quantité d'aliment ingéré avec les régimes contenant le $\mathrm{BE}(p<0,09)$. D'une façon générale, l'effet de l'Ex est non significatif pour tous les paramètres considérés.

Pour la période totale de croissance-finition, il n'y a pas de différence significative entre les performances de croissance (tableau III). La consommation journalière d'aliment et même la réduction de $5 \mathrm{j}$ de la durée d'engraissement ne sont pas significativement différentes entre les régimes. En période de croissance, au cours de laquelle les animaux ne sont pas rationnés, on observe une tendance à l'augmentation de l'ingestion et de l'indice de consommation 
Tableau III. Effet de l'addition de pulpe de betterave sur les résultats de croissance entre $25 \mathrm{~kg}$ et l'abattage.

\begin{tabular}{|c|c|c|c|c|c|c|c|}
\hline & \multicolumn{2}{|c|}{ Régimes } & \multicolumn{2}{|c|}{ Sexe } & \multicolumn{3}{|c|}{ Analyse statistique ${ }^{\prime}$} \\
\hline & $B N$ & $B N P$ & Castrés & Femelles & Régime & Sexe & ETR \\
\hline \multicolumn{8}{|l|}{ Période totale : 25 à $100 \mathrm{~kg}$} \\
\hline Poids initial $(\mathrm{kg})$ & 28,1 & 27,8 & 28,1 & 27,7 & 0,67 & 0,20 & 1,8 \\
\hline Poids final (kg) & 100,7 & 100,3 & 101,0 & 100,0 & 0,75 & 0,003 & 1,9 \\
\hline Durée (j) & 106 & 111 & 104 & 114 & 0,35 & 0,001 & 11 \\
\hline Aliment ingéré (g/jour) & 2130 & 2060 & 2170 & 2030 & 0,14 & 0,001 & 142 \\
\hline Gain de poids (g/jour) & 691 & 660 & 709 & 643 & 0,37 & 0,001 & 60 \\
\hline \multicolumn{8}{|l|}{ Indice de consommation } \\
\hline kg d'aliment/kilo gain PV & 3,10 & 3,13 & 3,07 & 3,17 & 0,54 & 0,001 & 0,15 \\
\hline MJ ED/kilo PV & 41,65 & 40,10 & 40,19 & 41,47 & 0,001 & 0,001 & 1,91 \\
\hline \multicolumn{8}{|l|}{ Croissance : 25 à $60 \mathrm{~kg}$} \\
\hline Aliment ingéré (g/jour) & 1730 & 1660 & 1760 & 1630 & 0,11 & 0,001 & 161 \\
\hline Gain de poids (g/jour) & 581 & 553 & 596 & 539 & 0,57 & 0,001 & 69 \\
\hline \multicolumn{8}{|l|}{ Indice de consommation } \\
\hline kg d'aliment/kilo gain PV & 3,01 & 3,02 & 2,97 & 3,05 & 0,11 & 0,01 & 0,19 \\
\hline MJ ED/kilo PV & 40,27 & 38,16 & 38,62 & 39,69 & 0,001 & 0,01 & 2,40 \\
\hline \multicolumn{8}{|l|}{ Finition : 60 à $100 \mathrm{~kg}$} \\
\hline Aliment ingéré (g/jour) & 2670 & 2650 & 2740 & 2590 & 0,21 & 0,001 & 107 \\
\hline Gain de poids (g/jour) & 843 & 820 & 870 & 793 & 0,29 & 0,001 & 61 \\
\hline \multicolumn{8}{|l|}{ Indice de consommation } \\
\hline kg d'aliment/kilo gain PV & 3,18 & 3,25 & 3,16 & 3,27 & 0,64 & 0,002 & 0,19 \\
\hline MJ ED/kilo PV & 42,96 & 41,94 & 41,67 & 43,19 & 0,07 & 0,001 & 2,52 \\
\hline
\end{tabular}

BN : blé normal, BNP : blé normal plus pulpe de betterave. 'Aucun effet significatif résiduel des régimes de postsevrage n'a été observé ; valeurs des moyennes ajustées et de la probabilité ; l'interaction régime*'sexe n'est pás significative ; ETR : écart type résiduel du modèle.

$(p<0,11)$ avec le régime $\mathrm{BN}$ mais qui ne se traduit pas sur le gain moyen quotidien (NS). En finition, les performances sont très similaires entre les deux régimes. En général, les animaux castrés ingèrent plus d'aliment que les femelles et ont une vitesse de croissance supérieure $(p<0,001)$, entrầnant une réduction de $10 \mathrm{j}$ de la durée totale d'engraissement et un indice de consommation plus faible $(p<0,01)$. Le calcul de l'indice de consommation énergétique montre que la croissance entre 25 et $60 \mathrm{~kg}$ de
PV est significativement $(p<0,001)$ moins coûteuse en ED pour les animaux recevant le régime contenant de la pulpe. La même tendance $(p<0,07)$ s'observe entre 60 et $100 \mathrm{~kg}$ de PV. Au cours des deux périodes et par conséquent sur l'ensemble de la durée de l'essai, les femelles ont eu un indice de consommation énergétique supérieur aux mâles castrés $(p<0,01)$.

Chez les porcelets utilisés pour la mesure de la digestibilité (tableau IV), on n'observe pas d'effet significatif du régime sur la crois- 
Tableau IV. Effet du blé extrudé et de la pulpe de betterave sur la croissance et la digestibilité fécale, iléale et post-iléale des constituants des régimes et la rétention azotée chez le porcelet.

\begin{tabular}{|c|c|c|c|c|c|c|c|c|}
\hline & \multicolumn{4}{|c|}{ Régimes } & \multicolumn{4}{|c|}{ Analyse statistique! } \\
\hline & $B N$ & $B E$ & $B N P$ & $B E P$ & $P b$ & $E x$ & $P b^{*} E x$ & ETR \\
\hline \multicolumn{9}{|l|}{ Période fécale } \\
\hline Poids initial (kg) & 7,71 & 7,59 & 7,71 & 7,60 & 0,99 & 0,60 & 0,99 & 0,60 \\
\hline Aliment ingéré (g/jour) & 223 & 223 & 234 & 234 & 0,07 & 0,90 & 0,95 & 15,3 \\
\hline Croissance (g/jour) & 150 & 130 & 167 & 151 & 0,09 & 0,09 & 0,82 & 30 \\
\hline \multicolumn{9}{|l|}{ Digestibilité $(\%)$} \\
\hline Matière sèche & 85,5 & 85,1 & 85,4 & 84,8 & 0,75 & 0,44 & 0,92 & 2,0 \\
\hline Énergie & 85,2 & 84,8 & 85,1 & 85,0 & 0,95 & 0,72 & 0,81 & 2,2 \\
\hline Azote & 81,8 & 82,1 & 83,2 & 82,7 & 0,41 & 0,95 & 0,68 & 3,2 \\
\hline Rétention azotée (g N/jour) & 3,8 & 3,8 & 4,2 & 4,0 & 0,18 & 0,53 & 0,60 & 0,6 \\
\hline \multicolumn{9}{|l|}{ Période iléale } \\
\hline Poids initial (kg) & 7,54 & 7,41 & 7,60 & 7,26 & 0,85 & 0,29 & 0,63 & 0,57 \\
\hline Aliment ingéré ( $\mathrm{g} /$ jour) & 247 & 248 & 244 & 250 & 0,95 & 0,30 & 0,35 & 7,0 \\
\hline Croissance (g/jour) & $128 \mathrm{ab}$ & $150 \mathrm{a}$ & $73 b$ & $7 \mathrm{lb}$ & 0,01 & 0,67 & 0,61 & 60 \\
\hline \multicolumn{9}{|l|}{ Digestibilité (\%) } \\
\hline Matière sèche & $70,3 a$ & $71,3 \mathrm{a}$ & $63,8 \mathrm{~b}$ & $64,7 \mathrm{~b}$ & 0,001 & 0,30 & 0,93 & 2,4 \\
\hline Énergie & $73,4 \mathrm{a}$ & $74,6 \mathrm{a}$ & $67,5 b$ & $68,3 b$ & 0,001 & 0,30 & 0,80 & 2,4 \\
\hline Azote & 72,2 & 72,9 & 68,1 & 68,4 & 0,06 & 0,81 & 0,93 & 5,1 \\
\hline Rétention azotée (g N/jour) & $3,7 \mathrm{a}$ & $3,8 \mathrm{a}$ & $2,4 b$ & $2,9 b$ & 0,001 & 0,13 & 0,40 & 0,5 \\
\hline \multicolumn{9}{|l|}{ Post-iléale } \\
\hline \multicolumn{9}{|l|}{ Digestibilité (\%) } \\
\hline Matière sèche & $52,4 b$ & $52,4 \mathrm{~b}$ & $61,0 \mathrm{a}$ & $56,8 \mathrm{ab}$ & 0,01 & 0,35 & 0,36 & 5,3 \\
\hline Énergie & $45,1 b$ & $44,8 \mathrm{~b}$ & $54,6 a$ & $51,4 a b$ & 0,02 & 0,56 & 0,63 & 7,3 \\
\hline Azote & 32,9 & 37,2 & 48,9 & 44,4 & 0,06 & 0,98 & 0,43 & 13,0 \\
\hline
\end{tabular}

BN : blé normal, BE : blé extrudé, BNP : blé normal plus pulpe de betterave, BEP : blé extrudé plus pulpe de betterave. ${ }^{~ V a l e u r s ~ d e s ~ m o y e n n e s ~ a j u s t e ́ e s ~ e t ~ d e ~ l a ~ p r o b a b i l i t e ́ ~ ; ~} \mathrm{~Pb}$; pulpe de betterave ; Ex : extrusion ; $\mathrm{Pb} *$ Ex : interaction pulpe de betterave*extrusion du blé ; ETR : écart type résiduel du modèle ; a, b, c : les valeurs moyennes qui ne sont pas affectées d'une même lettre sont significativement différentes à $p<0,05$ par le test de Student-Newman-Keuls.

sance pendant la période de collecte fécale. Toutefois, on retrouve la tendance $(p<0,09)$ déjà observée pour l'expérience zootechnique montrant une augmentation du gain de poids avec le régime contenant le BNP. En période de mesure iléale on observe environ $50 \%$ de réduction de la croissance $(p<0,01)$ lorsque les régimes contiennent de la pulpe de betterave.

\section{Digestibilité des composants du régime}

Chez le porcelet, la digestibilité fécale de la matière sèche, de l'énergie et de l'azote (tableau IV) n'est pas différente entre les régimes. Cependant, l'addition de pulpe entraîne une réduction d'environ $10 \%$ de la digestibilité iléale de la matière sèche et de l'énergie $(p<0,001)$ et la même tendance 
s'observe pour l'azote $(p<0,06)$. La digestibilité post-iléale, calculée par différence et sur les mêmes animaux, montre au contraire une amélioration significative de plus de $10 \%$ pour la matière sèche et l'énergie $(p<0,05)$; la même tendance se vérifie pour l'azote $(p<0,06)$, chez les animaux ayant reçu de la pulpe. La rétention azotée journalière n'est pas significativement modifiée durant la période fécale, alors qu'elle est réduite en période iléale $(p<0,001) \mathrm{du}$ fait de l'incorporation de la $\mathrm{Pb}$ dans l'aliment. Enfin, aucune des mesures ne permet de montrer une influence de l'Ex sur la digestibilité ou la rétention azotée.

\section{Caractéristiques corporelles et mesures sur la carcasse}

Les mesures d'épaisseur des tissus gras effectuées soit avec l'appareil à ultrasons sur l'animal vivant, soit sur la carcasse avec le dispositif optique Fat-O-Meater (fig 1) varient dans le même sens en fonction du régime. Elles montrent clairement que les animaux ayant reçu le régime contenant de la pulpe en croissance et finition sont toujours significativement moins gras, la différence étant déjà significative à $60 \mathrm{~kg}$ de PV au niveau du dos de l'animal $(p<0,01)$. Il faut également remarquer une différence des valeurs absolues entre les mesures effectuées à 1 semaine d'intervalle, soit à environ $90 \mathrm{~kg}$ de PV, soit sur la carcasse, pour des sites de mesure à peu près équivalents, au dos et au rein. Les différences entre régimes sont similaires mais, en valeurs absolues, celles mesurées au Fat-O-Meater sont toujours supérieures. À l'abattage, on a observé, pour un PV identique, un poids de carcasse chaude $(p<0,02)$ et un rendement $(p<0,05)$ significativement supérieurs chez les animaux qui ont reçu le régime sans pulpe (tableau V). Au contraire, on observe une augmentation du pourcentage de muscle de

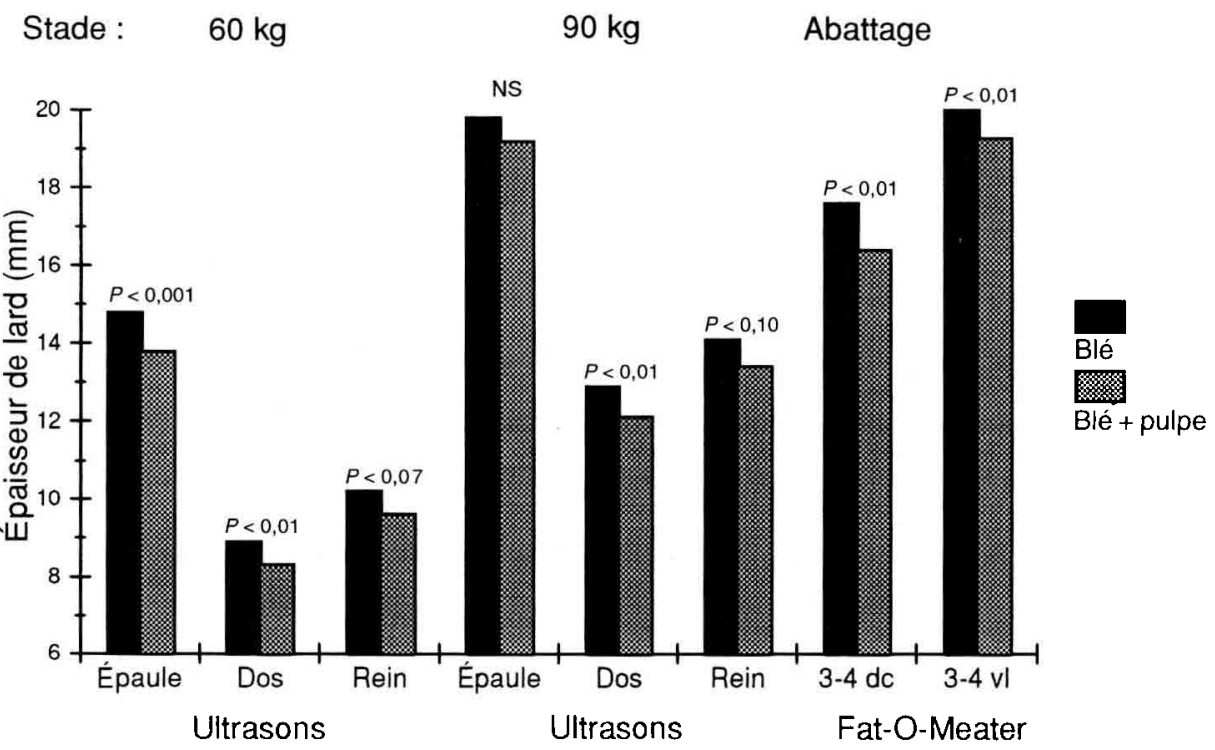

Fig 1. Effet de l'incorporation de pulpe de betterave dans l'aliment sur l'épaisseur du tissu adipeux selon le stade, le mode et le site de mesure (3-4 dc : 3-4 ${ }^{\mathrm{cs}}$ dernières côtes ; $3-4 \mathrm{vl}: 3-4^{\mathrm{es}}$ dernières vertèbres lombaires). 
Tableau V. Effet de l'incorporation de pulpe de betterave dans les régimes du porc en croissance finition sur les résultats d'abattage et de découpe parisienne normalisée de la demi-carcasse.

\begin{tabular}{|c|c|c|c|c|c|c|c|}
\hline & \multicolumn{2}{|c|}{ Régimes } & \multicolumn{2}{|c|}{ Sexe } & \multicolumn{3}{|c|}{ Analyse statistique $^{\prime}$} \\
\hline & $B N$ & $B N P$ & Castrés & Femelles & Régime & Sexe & ETR \\
\hline \multicolumn{8}{|l|}{ Mesures à abattage } \\
\hline Poids vif $(\mathrm{kg})$ & 100,7 & 100,3 & 101,0 & 100,0 & 0,51 & 0,003 & 2,0 \\
\hline Carcasse chaude $(\mathrm{kg})$ & 81,6 & 80,1 & 81,3 & 80,4 & 0,02 & 0,03 & 2,1 \\
\hline Rendement en carcasse $(\%)^{2}$ & 81,1 & 79,9 & 80,5 & 80,5 & 0,05 & 0,93 & 2,0 \\
\hline \multicolumn{8}{|l|}{ Épaisseurs (mm) } \\
\hline Lard dorsal (X2) & 20,9 & 19,3 & 21,7 & 18,6 & 0,03 & 0,001 & 2,7 \\
\hline Lard lombaire (X4) & 17,9 & 16,5 & 18,6 & 15,8 & 0,04 & 0,001 & 2,4 \\
\hline Longissimus dorsi (X5) & 53,1 & 52,1 & 51,2 & 53,9 & 0,33 & 0,05 & 6,1 \\
\hline Muscle $(\%)^{3}$ & 52,9 & 54,0 & 52,0 & 54,9 & 0,05 & 0,001 & 2,0 \\
\hline \multicolumn{8}{|l|}{ Mesures de découpe $(\mathrm{kg})^{+}$} \\
\hline Demi-carcasse & 37,4 & 36,6 & 37,1 & 37,0 & 0,07 & 0,83 & 1,3 \\
\hline Longe & 12,4 & 12,3 & 12,1 & 12,6 & 0,66 & 0,07 & 0,8 \\
\hline Jambon & 8,9 & 8,8 & 8,8 & 9,0 & 0,57 & 0,27 & 0,4 \\
\hline Hachage & 6,1 & 6,1 & 6,2 & 6,0 & 0,71 & 0,06 & 0,3 \\
\hline Poitrine & 4,2 & 4,0 & 4,2 & 4,0 & 0,08 & 0,10 & 0,3 \\
\hline Bardière & 3,8 & 3,4 & 3,9 & 3,3 & 0,007 & 0,001 & 0,4 \\
\hline Panne & 0,7 & 0,5 & 0,6 & 0,6 & 0,003 & 0,38 & 0,1 \\
\hline Tissu maigre ${ }^{5}$ & 20,3 & 20,2 & 19,8 & 20,1 & 0,81 & 0,03 & 1,1 \\
\hline Tissu gras ${ }^{5}$ & 8,6 & 7,6 & 8,6 & 7,5 & 0,001 & 0,001 & 0,8 \\
\hline
\end{tabular}

BN : blé normal, BNP : blé normal plus pulpe de betterave. ${ }^{\perp}$ Valeurs des moyennes ajustées et de la probabilité : l'interaction régime*sexe n'est pas significative ; ETR : écart type résiduel du modèle. ? Calculé à partir du PV avant abattage et du poids de la carcasse chaude. ${ }^{3}$ Calculé à partir de l'équation : $\mathrm{Y} 1=57,399-0,33 \mathrm{X} 2-0,441 \mathrm{X} 4$ $+0,193$ X5 (CCE Directive 50, 1989). ${ }^{4}$ Demi-carcasse gauche après 24 heures de ressuage et réfrigération. ${ }^{5}$ Calculé respectivement à partir des équations : Tissu maigre, $\mathrm{Y} 2=296,9-201,0$ Demi-carcasse froide $(\mathrm{kg})$ $+1,395$ longe $(\mathrm{g})+1,144$ jambon $(\mathrm{g})$; tissu gras, $\mathrm{Y} 3=-816+1,63$ bardière $(\mathrm{g})+1,43$ panne $(\mathrm{g})+0,52$ poitrine (g) (Desmoulin el al, 1988).

la carcasse $(p<0,05)$ avec l'addition de pulpe associée à la réduction de l'épaisseur du lard aux niveaux lombaire $(p<0,04)$ et dorsal $(p<0,03)$. L'épaisseur du muscle Longissimus dorsi ne varie pas significativement entre les traitements. L'effet sexe est significatif, sauf pour le rendement de carcasse. Ainsi les femelles présentent une carcasse significativement moins grasse qui se traduit par une différence dans le pourcentage de muscle $(p<0,001)$. À la découpe, on a observé une tendance à la réduction du poids de la demi-carcasse froide $(p<0,07)$ et des pièces grasses, poitrine $(p<0,08)$, bardière $(p<0,007)$ et panne $(p<0,003)$, chez les animaux ayant reçu le régime contenant de la pulpe. La longe et le jambon n'ont subi aucune influence du type de régime distribué aux animaux. On observe aussi que le poids des pièces grasses est plus faible et celui de la longe plus élevé chez les femelles que chez 
les mâles castrés. Finalement, le poids du tissu maigre (NS) et du tissu gras $(p<0,001)$ de la demi-carcasse calculés à partir du poids des morceaux de découpe confirment les estimations observées par les mesures linéaires.

\section{DISCUSSION}

\section{Effet de l'addition de pulpe sur les performances zootechniques}

L'incorporation de BE dans les régimes n'a pas amélioré la croissance des porcelets, ni en présence ni en l'absence de pulpe. Ces données confirment d'autres observations sur l'application de ce traitement technologique au blé (Pond et Maner, 1984), à l'orge (Fadel et al, 1988) et au maïs (Van der Poel et al, 1990 ; Herkelman et al, 1990). L'addition de $6 \%$ de $\mathrm{Pb}$, matière première riche en PNA, à l'aliment du porcelet semble favorable dès le sevrage puisque, pour une consommation d'aliment sensiblement identique, les performances de croissance et d'efficacité alimentaire sont améliorées au cours du premier âge. Au contraire, il paraît surprenant que l'addition de pulpe au régime contenant du $\mathrm{BE}$, donc un amidon très digestible, n'apporte pas la même amélioration. Nos observations confirment celles de Edwards et al (1991) et Longland et al (1994), qui n'ont pas trouvé de différence de vitesse de croissance après l'incorporation de $15 \%$ de pulpe dans un régime à base d'orge apportant déjà une certaine quantité de fibres. Ces résultats semblent indiquer que l'addition d'une quantité raisonnable de pulpe, ou de PNA à l'aliment de sevrage facilite le développement et l'adaptation du tube digestif du jeune à l'aliment concentré.

En période de croissance et de finition, les performances concernant le gain de poids sont modestes en raison d'un changement simultané d'aliment et de local et du fait de l'élevage en loge individuelle. Le gain moyen quotidien des animaux recevant le régime à base de pulpe n'a pas été réduit contrairement aux résultats de Chauvel et al (1975) obtenus avec un taux d'incorporation de $10 \%$ de pulpe. Chez le porc, on a fréquemment constaté (Henry, 1976) une augmentation de la quantité d'aliment ingérée en réponse à une concentration énergétique plus faible. La réduction de la teneur en ED avec l'incorporation de pulpe n'a pas eu cet effet sur l'ingestion, elle a même eu tendance à la réduire en période de croissance, contrairement aux observations de Chauvel et al (1975) avec des taux d'incorporation dans les régimes beaucoup plus forts.

\section{Conséquences digestives et nutritionnelles}

L'absence d'effet favorable du blé extrudé utilisé seul ou en association avec la pulpe sur la digestibilité est à souligner. De tels résultats ont déjà été observés par Van der Poel et al (1990) ou Herkelman et al (1990) avec le maïs extrudé, mais sont opposés aux résultats favorables observés sur la digestibilité iléale des constituants de l'orge (Fadel et al, 1988). Dans la présente expérience, le fait d'avoir un aliment sous forme de granulés a pu suffire à endommager la structure de l'amidon de blé, résultat déjà observé dans les aliments pour le porcelet (Aumaitre, 1976).

L'addition de $\mathrm{Pb}$ entraîne théoriquement (Noblet et al, 1989) une réduction de la teneur en ED des régimes, mais elle n'est pas confirmée par les mesures directes de digestibilité effectuées chez le porcelet au cours du premier âge. Ces données permettent de supposer, en dehors d'un remplissage du tube digestif, que les constituants pariétaux de la $\mathrm{Pb}$ sont partiellement digérés dans le tube digestif du porcelet, comme le montrent Longland et al (1994) ainsi que Graham et al (1986) et Chabeauti et al (1991) chez le porc en croissance. En outre, 
la pulpe semble favoriser l'activité de la flore microbienne du gros intestin du porcelet, en augmentant la digestibilité postiléale de tous les composants de la ration, comme l'avait démontré Drochner (1984) avec des pectines. Chez le porcelet, comme chez le porc en croissance, cette digestibilité post-iléale peut encore, à partir des AGV issus de la fermentation microbienne des fibres dans le gros intestin, contribuer à la couverture partielle des besoins énergétiques des animaux (Shi et Noblet, 1994b).

Le rapprochement des résultats sur les animaux en croissance avec ceux observés en cage de digestibilité permet de penser que la présence de pulpe réduit la digestibilité iléale de l'énergie et de l'azote mais qu'une partie des nutriments issus des fermentations du gros intestin est récupérée et absorbée. Les données de digestibilité postiléale témoignent à la fois du rôle et de l'efficacité de la digestion de certains constituants (fibreux ?) dans le gros intestin.

Cela explique, en conséquence, l'absence d'effet néfaste des régimes contenant de la pulpe sur les performances de croissance des animaux intacts, voire leur amélioration. En l'absence de compensation digestive issue du gros intestin, la croissance des porcelets porteurs d'anastomose iléorectale est donc fortement réduite car ils ne peuvent valoriser l'énergie produite sous forme d'acides gras volatils à partir des fermentations microbiennes.

\section{Conséquences sur les caractéristiques de la carcasse}

L'introduction de la pulpe dans la ration entraîne une baisse de 1,15 point du rendement et du poids de la carcasse en raison d'un poids plus élevé du contenu intestinal, soit une différence de $400 \mathrm{~g}$ en moyenne par animal. Ce résultat classique confirme les données de Anugwa et al (1989) observées lors de l'incorporation de farine de luzerne dans l'aliment. L'expression du rendement en carcasse par rapport au PV vide accroît la différence entre les deux groupes à 1,80 point. Ces observations confirment les résultats de Kay et al (1990) observés avec l'augmentation du contenu en $\mathrm{Pb}$ des régimes. Le résultat le plus intéressant concerne l'augmentation significative du pourcentage de muscle de la carcasse, observée parallèlement à la réduction de l'épaisseur du lard, en plein accord avec Chauvel et al (1975) et Kay et al (1990). Il n'existe pas de différence entre le poids des morceaux maigres de la carcasse alors que le poids des morceaux gras est réduit. L'absence d'effet du régime sur le poids du jambon et de la longe montre enfin qu'une réduction modérée de la teneur en ED du régime par l'addition de pulpe réduit le degré d'adiposité à l'abattage, mais n'affecte pas la proportion de muscle dans la carcasse, et cela quel que soit le sexe des animaux. Les différences observées in vivo à partir des mesures d'épaisseur du lard (fig 1) montrent, à la fois à 60 et à $90 \mathrm{~kg}$ de PV, une propension des animaux recevant de la pulpe à déposer moins de gras, confirmant les caractéristiques de la carcasse observées à l'abattage. L'absence d'effet du taux de fibres alimentaires sur la rétention azotée journalière soulignée par Low (1993) chez le porc en croissance peut expliquer le fait que la quantité de tissu maigre de la carcasse ne soit pas affectée par la présence de pulpe dans la ration.

\section{CONCLUSION}

L'addition d'une faible proportion, $6 \%$, de pulpe de betterave à la ration dès le sevrage permet uniquement une amélioration des performances de croissance des porcelets au premier âge, probablement liée à une action favorable des PNA sur la fonction de digestion. En outre, l'utilisation de blé extrudé ne présente pas d'avantage comparable pour justifier son incorporation dans 
les aliments du porcelet. L'incorporation de la pulpe de betterave dans la ration n'a pas réduit significativement la vitesse de croissance chez le porc en croissance-finition, mais elle entraîne une réduction de l'épaisseur et du poids des pièces grasses sans modifier le poids des pièces maigres, ce qui se traduit par une augmentation du pourcentage de muscle. Le système de classement de la carcasse fondé sur le pourcentage de muscle peut ainsi contribuer à un résultat économique favorable dans le cas de la formulation de régimes supplémentés en pulpe pendant la période de croissancefinition.

\section{REMERCIEMENTS}

Nous tenons à remercier le programme Praxis XXI de la JNICT (Junta Nacional de Investigação Científica e Tecnológica, Portugal) pour le soutien financier accordé à $\mathrm{R}$ Lizardo, ainsi que le personnel de la station de recherches porcines pour sa collaboration à l"expérimentation, G Conseil, M Lefèvre, M Massard, H Demay, M Besnard, P Dubois, P Bodinier, M Alix et JP Prigent.

\section{RÉFÉRENCES}

AOAC (1990) Official methods of analysis. 15th ed, AOAC, Washington DC, $1230 \mathrm{p}$

Anugwa FO, Varel VH, Dickson JS, Pond WG, Krook LP (1989) Elfects of dietary fiber and protein concentration on growth, feed efficiency, visceral organ weights and large intestine microbial populations of swine. $J$ Nutr 119,879-886

Aumaitre A (1976) Évaluation de divers traitements technologiques aux céréales. IV. Influence du floconnage et de l'expansion de l'orge et du maïs sur les performances des porcelets sevrés à $21 \mathrm{j}$ : effets sur la digestibilité des éléments de la ration. Ann Zootech 25, 41-51

Björck I, Asp NG (1983) The effects of extrusion cooking on nutritional value. A literature revicw. $J$ Food Eng 2, 281-308

Bolduan G, Jung H, Schnabel E, Schneider R (1988) Recent advances in the nutrition of weaner piglets. Pig News Information 9, 381-385

Bourdon D, Henry Y (1985) Effets de la supplémentation en lysine industrielle sur les performances de croissance chez le porc : comparaison de deux formes d'apport (HCl et sulfate). Journées Rech Porcine en France 17, 371-381

CCE (1989) Décision de la Commission 89/50/CEE relative à l'autorisation des méthodes de classement des carcasses de porc en France. J Off CCE $\mathrm{n}^{\circ}$ L 20 du 25/1/89, 27-30

Chabeauti E, Noblet J, Carré B (1991) Digestion of plant cell walls from four different sources in growing pigs. Anim Feed Sci Technol 32, 207-213

Chauvel J, Villain-Guillot J, Bourdon D (1975) Valeur énergétique de pulpes de betteraves déshydratées et utilisation par le porc en croissance-finition. Journées Rech Porcine en France 7, 53-60

Desmoulin B, Ecolan P, Bonneau M (1988) Estimation de la composition tissulaire des carcasses de porcs : récapitulatif de diverses méthodes utilisables en expérimentation. INRA Prod Anim 1, 59-64

Drochner W (1984). The influence of changing amounts of crude fibre and pectic components on prececal and postileal digestic processes in the growing pig. I Anim Physiol Anim Nutr, suppl no 14

Edwards SA, Taylor AG, Harland JI (1991) The inclusion of sugar-beet pulp in diets for early-weaned piglets. Anim Prod 52, 131 (Abstr)

Eidelsburger U, Kirchgessner M, Roth FX (1992) Influence of fumaric acid, hydrochloric acid, sodium formate, tylosin and toyocerin on daily weight gain, feed intake, feed conversion rate and digestibility. 1. Investigations about the nutritive efficacy of organic acids in the rearing of piglets. J Anim Physiol Anim Nutr 68, 82-92

Fadel JG, Newman CW. Newman RY, Graham H (1988) Effects of extrusion cooking of barley on ileal and fecal digestibilities of dietary components in pigs. Can J Anim Sci 68, 891-897

Graham H, Hesselman K, Aman P (1986) The influence of wheat bran and sugar-beet pulp on the digestibility of dietary components in a cereal-based pig diet. $J$ Nutr 116,242-25l

Henry Y (1976) Prediction of energy values of feeds for swine from fiber content. In: Feed Composition, Animal Nutrient Requirements and Computerization of Diets (PV Fonnesbeck, LE Harris, LC Kearl, eds) Utah Agr Exp Sta, Utah State University, Logan, Utah, USA, 270-286

Herkelman KL, Rodhouse SL, Veum TL, Ellersieck MR (1990) Effect of extrusion on the ileal and fecal digestibilities of lysine in yellow corn in diets for young pigs. J Anim Sci 68, 2414-2424

Inra (1989) L'Alimentation des animaux monogastriques: porc, lapin, volailles. Inra ed, Paris

Kay RM, Simmins PH, Harland JL (1990) The use of molassed sugar-beet feed in growing pig diets and the effect of inclusion rates on subsequent performance. Anim Prod 50, 154 (Abstr) 
Kelly D, O'Brien JJ, McCracken KJ (1990) Effect of creep feeding on the incidence, duration and severity of postweaning diarrhoea in pigs. Res Vet Sci 49. 223-228

Laplace JP, Souffrant WB, Hennig U. Chabeauti E. Fevrier C (1994) Measurement of precaccal dictary protein and plant cell wall digestion in pigs ; comparison of four surgical procedures for ileorectal anastomosis. Livest Prod Sci 40, 313-328

Lizardo, Peiniau J, Aumaitre A (1995) Effect of sorghum on performance, digestibility of dietary components and activities of pancreatic and intestinal enzymes in the weaned piglet. Anim Feed Sci Technol $56,67-82$

Longland AC, Carruthers J, Low AG (1994) The ability of piglets 4 to 8 wecks old to digest and perform on diets containing 2 contrasting sources of nonstarch polysaccharide. Anim Prod 58, 405-410

Low AG (1993) Role of dietary fibre in pig diets. In : Recent advances in animal nutrition (Haresign W, Cole DJA, eds) Butterworths, 87-112

Low AG, Carruthers JC, Longland AC, Harland JI (1990) Performance and digestibility of non-starch polysaccharides in cereals or sugar-bect pulp in pigs of 3 to 8 weeks. Brit Soc Anim Prod. Winter Meeting, Scarborough, Paper no 149

Noblet J, Fortune H, Dubois S, Henry Y (1989) Nouvelles méthodes d'estimation des teneurs en énergie digestible, métabolisable et nette des aliments pour le porc. Inra Éditions Paris

Partridge GG, Gill BP (1993) New approaches with pig weaner diets. In : Recent adrances in animal mutrition (PC Garnsworthy, DJA Cole, eds) Nottingham University Presis, 221-247

Peiniau J, Aumaittre A, Lebreton Y (1996) Influence of physical properties of dietary protein on fecal and ileal digestibility of nitrogen and activity of pancreatic enzymes in the weaned piglet. Livest Prod Sci 45, 197-208

Pond WP, Maner JH (1984) Processing and its effect on Nutritive Value. In : Swine Production and Nutrition (WG Pond, JH Maner, eds) AVI Publishing Co. Westport. Connecticut, 584-603

SAS (1990) User's guide: Statistics. Statistical Analysis Systems Institute, Inc, Cary, NC

Shi XS, Noblet J (1994a) Digestible and metabolizable energy values of ten feed ingredients in growing pigs fed ad libitum and sows fed at maintenance level; comparative contribution of the hindgut. Anim Feed Sci Technol 42, 223-236

Shi XS, Noblet J (1994b) Effect of body weight and feed composition on the contribution of hindgut to digestion of energy and nutrients in pigs. Livest Prod Sci 38, 225-235

Van der Poel AFB, Den Hartog LA, Van Stiphout WAA, Bremmers R, Huisman J (1990) Effects of extrusion of maize on ileal and faecal digestibility of nutrients and performance of young piglets. Anim Feed Sci Technol 29, 309-320

Van Soest PJ, Wine RH (1967) Use of detergents in the analysis of fibrous feeds. IV. Determination of plant cell wall constituents. J Ass Off Anal Chem 50 , 50-55 